\section{Hipatia Press}

www.hipatiapress.com

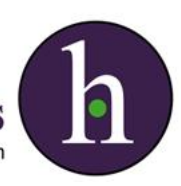

Instructions for authors, subscriptions and further details:

http://rise.hipatiapress.com

\title{
50 Años Después del Informe Coleman. Las Actuaciones Educativas de Éxito sí Mejoran los Resultados Académicos
}

Ramón Flecha ${ }^{1}$

Nataly Buslon ${ }^{1}$

1) Universidad de Barcelona, Spain

Date of publication: June $25^{\text {th }}, 2016$

Edition period: June 2016-October 2016

To cite this article: Flecha, R., Buslon, N. (2016). 50 años después del Informe Coleman. Las actuaciones educativas de éxito sí mejoran los resultados académicos. International Journal of Sociology of Education, 5(2), 127-143. doi: 10.17583/rise.2016.2087

To link this article: http://dx.doi.org/10.17583/rise.2016.2087

\section{PLEASE SCROLL DOWN FOR ARTICLE}

The terms and conditions of use are related to the Open Journal System and to Creative Commons Attribution License (CC-BY) 



\section{Years after the Coleman Report. Successful Educational Actions Improve Academic Results}

Ramón Flecha

Universidad de Barcelona
Nataly Buslon

Universidad de Barcelona

(Received: 9 May 2016; Accepted: 25 May 2016; Published: 25 June 2016)

\section{Abstract}

The report Equalitity of Educational Opportunity (EEO) by James S. Coleman in 1966, stated that school had little effect on educational equality, highlighting the socio-economic and ethnic differences as key elements for learning. These assumptions inspired decades of research on the role of school in academic performance, leading to a reproductionist discourse that denied the possibility of greater equality. Authors like H. Levin (1972), or the Black Response responded immediately to the statistical failures and negative social consequences of this report and similar ones like that of C. Jencks (1972). Taking into account all the variables contemplated by Coleman, this article focuses on the European program of scientific research, which demonstrated that developing successful educational actions (SEAs) or not varies very significantly the academic results.

Keywords: Coleman report, reproduction, inequalities, Jencks, Levin, Black response, Freire, Ferrer i Guàrdia. 


\section{Años Después del Informe Coleman. Las Actuaciones Educativas de Éxito sí Mejoran los Resultados Académicos}

Ramón Flecha

Universidad de Barcelona
Nataly Buslon

Universidad de Barcelona

(Recibido: 9 de Mayo 2016; Aceptado: 25 de Mayo 2016; Publicado: 25 Junio 2016)

\section{Resumen}

El informe Equalitity of Educational Opportunity (EEO) de James S. Coleman en 1966, señaló el escaso efecto de la escuela en la igualdad educativa, marcando el contexto socioeconómico y las diferencias étnicas como elementos claves para el aprendizaje. Estos supuestos inspiraron décadas de investigación sobre el rol de la escuela en el rendimiento académico, generando un discurso reproduccionista que negaba la posibilidad de mayor igualdad. Ya autores como H. Levin (1972), o los de la Black Response respondieron inmediatamente a los fallos estadísticos y las negativas consecuencias sociales de ese informe y otros análogos como el de C. Jencks (1972). Este artículo presenta cómo el programa de investigación científica europeo ha demostrado que la realización de actuaciones educativas de éxito (AEE) varía muy significativamente los resultados académicos, superando así todas las variables deterministas consideradas por Coleman.

Palabras clave: Informe Coleman, reproducción, desigualdades, Jencks, Levin, Black response, Freire, Ferrer i Guàrdia 

0 años después de la publicación del informe Equality of Educational Opportunity Study, más conocido como el Informe Coleman, volvemos a recuperar el debate sobre los erróneos supuestos a los que llegó, atribuyendo al contexto socioeconómico y al origen étnico la categoría de determinantes para el aprendizaje y los resultados educativos. Pero a pesar de que esos supuestos se rebatieran al poco tiempo por multitud de autores coetáneos a Coleman, este informe y otros estudios análogos han inspirado décadas de un discurso reproduccionista que ha tenido consecuencias muy negativas al negar la posibilidad de más igualdad a los colectivos más desfavorecidos. En la actualidad proyectos como INCLUDED, financiados por del Programa Marco de Investigación Europea, siguen demostrando como el nivel socioeconómico y el origen étnico no son determinantes académicos cuando en la escuela se implementan actuaciones educativas de éxito.

El informe Equality of Educational Opportunity Study fue financiado por el United States Department of Health, Education, and Welfare, tenía la finalidad de evaluar la igualdad de oportunidades educativas en los niños y niñas según su origen étnico, religión y nacionalidad a través de la encuesta llevada a cabo por el National Center for Education Statistics en una muestra de 645.000 niños y niñas de un total de 4.000 escuelas de Estados Unidos. El error grave de sus conclusiones radicó en confundir lo que es una correlación entre variables, como el entorno socioeconómico y las aspiraciones familiares, con una relación causal.

Las conclusiones del Informe Coleman trajeron consigo serias repercusiones. Fortalecieron el debate que creaba un destino determinista para los niños y las niñas de los grupos más desfavorecidos y, por lo tanto, sirvieron de pretexto para desacreditar el apoyo a políticas públicas que fueran destinadas a la mejora educativa de la población más vulnerable. El Informe Coleman se había orientado a desacreditar una década de intervención educativa en apoyo a los hijos e hijas de los negros.

\section{Inspiración de Otros Estudios Reproduccionistas: The School and Equal Opportunity (Bane \& Jenkcs, 1972) e Inequality (Jencks, 1973)}

Estos supuestos inspiraron décadas de investigación sobre el rol de la escuela en el rendimiento académico, generando un discurso 


\section{Flecha \& Buslon-Actuaciones Educativas de Éxito}

reproduccionista que negaba que la escuela pudiera contribuir a la superación de desigualdades. Al Informe Coleman se añadieron otros estudios que no solo perjudicaron a los niños y niñas negros sino también a los hijos e hijas de las familias con pocos recursos económicos. Entre los trabajos más influyentes se encontraban los estudios The School and Equal Opportunity de Mary Jo Bane y Cristopher Jenkcs (1972) e Inequality de Jencks y colaboradores (1972). Jencks y sus colegas defendieron que el Informe Coleman, pese a correcciones que debían señalarse en consideración a los procedimientos, revelaba conclusiones que llevaban a afirmar que las reformas escolares son irrelevantes para el logro de la igualdad. Una de las conclusiones de estos estudios tuvo una gran influencia (Bane \& Jencks, 1972, p. 37): "las diferencias entre las escuelas tienen un efecto muy pequeño en lo que ocurre en los estudiantes después de la graduación”. Ello implica que "la reforma escolar no tendrá nunca una significativa influencia en el grado de desigualdad entre los adultos" (Bane \& Jencks, 1972, p. 41). Según estos autores esa era la conclusión de un estudio de cuatro años sobre un amplio conjunto de datos sociológicos y económicos. Adoptar ese tipo de supuesto lleva a consecuencias terribles y muy reaccionarias. Primero, ese determinismo socioeconómico y étnico acaba llevando a la supresión o a debilitar la financiación a programas dirigidos a la salida de la desigualdad de los grupos sociales más desfavorecidos. Segundo, acaba destruyendo lo esfuerzos de profesorado y familias para mejorar la educación de los niños y niñas, haciendo que se acabe cumpliendo la fatal expectativa que se espera de esos colectivos. Tercero, quita todo valor a las ciencias sociales y de la educación, así como a la innovación educativa que sigue trabajando por una educación que supera esas desigualdades.

Las conclusiones tanto del Informe Coleman como las extraídas por Jencks y colaboradores tuvieron una fuerte influencia en la sociología de la educación, aún incluso para autores que eran contrarios a los trabajos cuantitativos, con la excusa que eran las únicas investigaciones coherentes dentro de esta disciplina. Pero no eran los únicos datos y análisis disponibles y las evidencias de sus incorrecciones fueron pronto puestas de manifiesto por diferentes autores y el propio Jencks no tardó en reconocerlas. Volvemos a recoger, ahora en este artículo, algunas de las respuestas que rebatieron los errores y fallos estadísticos de los trabajos de Coleman y de los de Jencks y colaboradores. 


\section{Respuestas al Objetivismo Norteamericano. El Progresivo Cambio de Jencks}

En 1972 Henry Levin ya resaltó las diferencias entre Inequality y otros estudios. En su línea habitual de trabajo más riguroso Levin analizó y comparó diferentes estudios que medían la influencia del nivel educativo y los ingresos futuros sacando a la luz los diferentes resultados entre estos. En el estudio de Jencks se afirmaba que la escolarización sólo tenía una influencia en los ingresos futuros de un $12 \%$ a un $15 \%$. Mientras que otros estudios habían encontrado que esta influencia podía llegar hasta el $50 \%$. Levin también visibiliza los fallos en el tratamiento estadístico de Inequality al omitir los lugares de residencia, de gran importancia por las diferencias de coste de la vida entre ellos. De las conclusiones de Levin queda claro que se requieren unos mínimos niveles educativos para una participación económica completa.

En la primavera de 1973 se publica un monográfico en la Harvard Educational Review donde se recogen algunos de los artículos que más impacto han tenido en desvelar los fallos de los trabajos de Jencks y colaboradores así como los del Equalitity of Educational Opportunity de Coleman.

Entre estos, el artículo de Lester C. Thurow (1973), publicado en el monográfico de la Harvard Educational Review, cuestiona las conclusiones de Inequality. Mientras estudios como los de Jencks y otros ponían el foco en las diferencias individuales señalando que estas eran mucho mayores que las diferencias entre grupos (por ejemplo, negros versus blancos), los teóricos del capital humano centraron la atención en la influencia de la inversión educativa para el grupo. Thurow señaló que ambas eran líneas de investigación que no son excluyentes pero que parten de supuestos diferentes y que con actuaciones educativas sí se podían combatir las diferencias entre grupos.

Ante el desarrollo del Informe Coleman y las obras de Jencks y colaboradores un grupo de científicos sociales negros iniciaron en octubre de 1972 la Black response, que fue publicada también en el mismo monográfico de la Harvard Educational Review. Esta respuesta puso de manifiesto que este tipo de trabajo compartía el dudoso honor de apoyar a opositores de la 


\section{Flecha \& Buslon-Actuaciones Educativas de Éxito}

reforma social cuyo objetivo era disminuir el apoyo presupuestario a las reformas iniciadas en los años sesenta. Durante los primeros años de esa década se llevaron a cabo importantes reformas sociales y educativas que partían de valiosas aportaciones y apoyos desde las ciencias sociales. Pero no tardaron las reacciones en contra de estas aportaciones que alcanzaron una rápida difusión con el debate abierto por los trabajos de Coleman y los de Jencks. Estos aportaban una cobertura dentro de las ciencias sociales a quienes les interesaba sostener que eran inútiles los esfuerzos que iban dirigidos a que los negros y las clases sociales bajas tuvieran la oportunidad de mejorar sus vidas.

Los autores de la Black response señalaban directamente este hecho: "premeditada o impremeditadamente, el libro de Jencks y sus trabajos hermanos harán mucho más fácil el deshacerse de los avances de la igualdad de oportunidades y los derechos civiles de los años sesenta" (Edmonds et al., 1973, p. 80). La Black response denunciaba que siempre que había un avance en la igualdad de los negros y los blancos con escasos recursos surgían supuestos estudios desde las ciencias sociales que cuestionaban la viabilidad de ese avance hacia la igualdad. Esta denuncia, a pesar de las décadas que hace que fue publicada, sigue siendo un tema de autorreflexión clave a incorporar en las ciencias sociales y, como parte de estas, en la sociología de la educación. Por qué cuándo grupos que han sufrido siglos de discriminación y segregación logran avances para una mayor inclusión social e igualdad, como sería el caso del pueblo gitano en España, siempre aparece algún académico que sin ninguna rigurosidad científica se atreve a desprestigiar los estudios y esfuerzos desde las ciencias sociales y la comunidad educativa que están trabajando por esa igualdad. En este mismo monográfico de RISE uno de los artículos responde a esta importante cuestión para la sociología de la educación.

Jencks en el mismo número de la Harvard Educational Review ya inicia su rectificación ante la posición que previamente había defendido y afirma que el mejor mecanismo para romper el círculo vicioso de la desigualdad entre grupos es la reforma educativa. Consideró el hecho de que los niños y niñas de hogares desfavorecidos no recibían de sus padres los debidos conocimientos. Ello le llevó a proponer actuaciones concretas para superar esa desigualdad como que estos niños y niñas tendrían que asistir a las mismas escuelas que los niños y niñas de clase media, que habría que 
proporcionarles programas extra compensatorios y que se tendrían que abrir las escuelas a la participación de las familias, o una combinación de estas tres actuaciones. Finalmente, en 1979 mediante un nuevo libro, Who Gets Ahead?, Jencks modificó sus cálculos reconociendo una mayor importancia de la escolarización en los ingresos futuros. En sus posteriores publicaciones cada vez aparece una mayor preocupación por la desigual distribución del crecimiento educativo y sus repercusiones sociales.

\section{Bowles y Gintis también Transformaron su Posicionamiento Inicial}

A pesar de las repercusiones en el modelo de la reproducción que tuvieron el Informe Coleman y los trabajos de Jencks y colaboradores, estos no desarrollaron en sí una teoría de la sociología de la educación. Esta teoría fue desarrollada posteriormente por sus colegas de Harvard Samuel Bowles y Herbert Gintis (uno de los autores de Inequality). Estos autores recogen los datos de Coleman y Jencks en una teoría de la correspondencia entre la educación y la producción que acaban desarrollando en profundidad en su libro Schooling in Capitalist America (1976). Bowles y Gintis se centran en el concepto de correspondencia, que establecía una estrecha dependencia del mundo de la escuela respecto del de la producción: "consideramos que el sistema educativo contribuye a la integración de la juventud en el sistema económico a través de la correspondencia estructural entre sus relaciones sociales y las de la producción" (Bowles \& Gintis, 1976, p. 131). Esta teoría ha recibido muchas críticas por reduccionista y economicista al ignorar otras formas de opresión como el sexismo o el racismo. La teoría de la correspondencia compartía elementos clave de su marco teórico con el modelo de la reproducción. Para esta teoría las reformas sociales y educativas tampoco tenían ninguna trascendencia.

Levin rebatió esos primeros argumentos de Bowles y Gintis poniendo de manifiesto que si la inversión en reformas no puede mejorar el futuro de los niños y niñas de los grupos más desfavorecidos ¿por qué se invertía entonces mucho más en la escolarización de los ricos? No era casualidad así recogía ya en 1972 esa incongruencia: "en el contexto de Bowles \& Gintis el hecho de que la sociedad gaste alrededor del doble en la escolarización de los ricos que de los pobres no es más que un inocuo accidente" (Levin, 1972, p. 51). La orientación fundamental estaba marcada por el estructuralismo marxista, 


\section{Flecha \& Buslon-Actuaciones Educativas de Éxito}

obviando a autores como Gramsci que han servido de base para el desarrollo de una sociología de la educación de orientación no estructuralista. Más tarde Bowles y Gintis (1988) acabaron reconociendo la influencia de Althusser.

Schooling in Capitalist America de Bowles y Gintis (1976) pasa a ser conjuntamente con los trabajos de Coleman y Jencks una de las obras más influyentes en el modelo de la reproducción. Este modelo ha realizado contribuciones a la sociología de la educación como el análisis de las desigualdades sociales, económicas y culturales en relación con la escuela, la escuela y movilidad social, la escuela como aparato ideológico del estado, o críticas a posteriori de los sistemas educativos en las diferentes épocas y países. Pero no puede explicar el cambio educativo. Berstein (1990) señaló cómo el modelo de la reproducción no ofrece unas categorías desde las que se expliquen cuándo hay lo mismo y cuándo hay cambio: "el cambio queda relegado al cambio de milenio. El cambio acontece cuando hemos logrado la transformación de la base social de la producción" (Bernstein 1990, p. 17). Lo que puede extraerse de los análisis del modelo de la reproducción es que las actuaciones de todos los agentes que intervienen en el ámbito educativo no variarán un resultado que está determinado por las estructuras de las que esos agentes dependen.

La afirmación tomada desde el modelo de la reproducción de que "la escuela no es responsable de las desigualdades sociales y no las cambia" es errónea, conservadora y muy negativa tanto para los sectores sociales que sufren la exclusión social como para la propia sociología de la educación. Esa frase surge de una mala traducción de un artículo de Bane y Jenks que no realizaba esa afirmación. Pero los reproduccionistas más serios no han seguido ese camino, ya se han señalado las rápidas rectificaciones de Jencks. También Bowles y Gintis fueron transformando su posicionamiento y a finales de los ochenta afirmaron que "nuestros lectores no deben tomar Schooling in Capitalist America como una teoría comprensiva de la escolarización, sino como una teoría de la vinculación de la educación y economía" (Bowles \& Gintis, 1988, p. 236). Ambos han ido cambiando su marco teórico abandonando el estructuralismo marxista por una visión de la sociedad como un conjunto de juegos, pretendiendo asumir una concepción que recoja tanto la acción como la estructura. 


\section{La Mediocridad de Althusser y de Quienes le Siguieron}

Althusser reconoció la mediocridad de su estructuralismo marxista y el modelo de la reproducción a que dio lugar. Ha llegado incluso a reconocer que escribió Para leer el Capital (1969), sin haber leído él mismo El Capital: "No sabía casi nada de la historia de la filosofía y casi nada de Marx (de quien ciertamente había estudiado sus libros jóvenes, pero de quien solo había leído seriamente el volumen I de El Capital). Raymond Aron no estaba totalmente equivocado hablando sobre mí y sobre Sartre de "marxismo imaginario" (Althusser, 1992, pp. 196-197).Volvemos a recordar aquí el hecho de que muchos autores reproduccionistas, especialmente en España, no solo no leyeron nunca El Capital sino tampoco Para leer el Capital, conformándose con textos de divulgación como el de una alumna de Althusser, Marta Hanecker. Por lo tanto, no conocían seriamente la obra de Marx, pero se apoyaban en las construcciones imaginarias de su obra que habían leído en otros textos u oído en conversaciones de café para afirmar la base científica del modelo de la reproducción y la inocencia humanista de los autores como Freire, el grupo de científicos afroamericanos de la Black response y movimientos sociales que trabajaban por transformaciones igualitarias desde la educación. Por ello, no es extraño que esa actitud les llevara a abrazar el Informe Coleman o The Schools and Equal Opportunity de Bane y Jencks, e ignorarlo rechazar tanto las críticas a esos trabajos como las propias rectificaciones de los autores. En este contexto se entiende por qué desde la sociología de la educación española se ha ignorado u ocultado el monográfico que en la primavera de 1973 se publicó en la Harvard Educational Review.

Una de las causas de esa resistencia se debe a que la perspectiva del modelo de la reproducción se ha presentado frecuentemente en nuestro país con una pretensión totalizante, como la única sociología de la educación posible. Ello ha llevado a que muchas décadas después aún se siga explicando lo que permanece, lo que reproduce la desigualdad, obviando o desprestigiando las actuaciones educativas que sí están produciendo cambio y que están demostrando que los hijos y las hijas de los inmigrantes, de las familias gitanas y de familias con bajos niveles socioeconómicos pueden tener éxito educativo.

Esa incapacidad del modelo de la reproducción para estudiar el cambio 


\section{Flecha \& Buslon-Actuaciones Educativas de Éxito}

ha sido clave para que la sociología actual se aparte del estructuralismo y parta de concepciones duales de la sociedad.

\section{Paulo Freire y la Escuela Moderna de Ferrer i Guàrdia}

Muchas son las aportaciones realizadas desde la sociología internacional a la concepción dual de la sociedad y de la educación que han ofrecido multitud de evidencias que han rebatido los supuestos deterministas del Informe Coleman y el modelo de la reproducción. Entre ellas, los conceptos del mundo de la vida de la Teoría de la Acción Comunicativa de Habermas (1987), la agencia humana en la Teoría de la Estructuración de Giddens (1990a; 1990b), los análisis de Apple (1986) sobre los procesos de resistencia que se desarrollan en la escuela, el análisis de los productores culturales de Willis (1990), los análisis de Giroux (1992) sobre la estructura, la agencia humana y el rol dentro de la política cultural, entre otros.

Pero queremos aquí hacer mención a Paulo Freire y Francesc Ferrer i Guàrdia como dos grandes pedagogos que han sido silenciados $\mathrm{y}$ desprestigiados desde la mediocridad intelectual de quienes impusieron en España una sociología de la educación seguidora del modelo de la reproducción. Una de las consecuencias de este silenciamiento ha sido que se ha prescindido de las aportaciones de estos dos autores en las diferentes reformas educativas de este país.

Freire ha sido el principal autor de la educación y referencia de Apple y Giroux, dos de los más importantes sociólogos de la educación en la actualidad. Ferrer i Guàrdia fue el creador de la Escuela Moderna (19011906), una de las muy pocas experiencias educativas que en España, 50 años antes que el Informe Coleman, tuvo un importante impacto internacional.

La perspectiva dialógica de la educación de Freire (1970) ya en los años sesenta parte del diálogo entre toda la comunidad incluyendo a padres, madres, familiares, voluntariado, profesorado y alumnado como una de las claves que influyen en al aprendizaje. Las actuaciones educativas que han recogido esta perspectiva están demostrando que análisis como los de Coleman o Jencks no pueden explicar cómo llegan a conseguir éxito educativo para todos $\mathrm{y}$ todas independientemente de su nivel socioeconómico y su origen étnico o cultural. La perspectiva dialógica de Freire lleva a plantearse una acción conjunta y consensuada entre todos los 
agentes del aprendizaje que interactúan con el alumnado; así como a la formación de todos estos agentes y no sólo restringida al profesorado. Freire, como seguidor de Vygotsky (1979), tenía clara la relación entre desarrollo cognitivo y entorno sociocultural y su propuesta de transformar el entorno para provocar este desarrollo. Plantea que "no somos seres de adaptación sino de transformación" (Freire, 1997) y coincidía plenamente con Vygotsky en que lo importante es transformar el contexto para lograr una plena alfabetización de las comunidades.

Esta perspectiva transformadora es la que siguen las actuaciones que aplican a la práctica una evidencia: que todas las niñas y los niños de familias con bajos niveles socioeconómicos, hijas e hijos de inmigrantes o pertenecientes a minorías culturales que han sufrido siglos de segregación lograrán éxito académico si no sólo se transforman las interacciones que se dan en el aula sino también las que se dan en casa y en la calle.

Todas las reformas que han cogido como modelo los supuestos a los que llegaba el Informe Coleman, los trabajos de Jencks u otras obras del modelo de la reproducción han llevado a que en las escuelas a las que van las niñas y los niños de niveles socioeconómicos más elevados se concentren aprendizajes mucho más elevados que los de los barrios más populares. Estas reformas orientadas a la adaptación a la diversidad han aumentado las desigualdades sociales y educativas.

La obra de Ferrer i Guàrdia siempre tuvo enemigos, tanto históricos como actuales. Solo algunas pocas personas desde los movimientos de renovación pedagógica y la universidad han reivindicado la vigencia de sus aportaciones. El propio Freire en una de sus visitas a Barcelona nos preguntó por la persecución y la campaña de difamación que sufrió y que finalmente acabó con su detención y ejecución, así como con el cierre de la Escuela Moderna unos años antes. Pero no vamos a entrar aquí en ese aspecto. Un artículo brillante de sociólogas y pedagogas españolas (Garcia-Yeste, Redondo-Sama, Padrós \& Melgar, 2016), recientemente publicado en la revista Teachers College Record de la Columbia University, analiza el contexto en el que se dieron esos ataques y los objetivos de eliminar tanto a la persona de Ferrer i Guàrdia como su obra.

Pero a pesar de ello, sus aportaciones a través de la Escuela Moderna (1901-1909) han influido a una infinidad de proyectos en diferentes países del mundo. Ferrer i Guàrdia con La Escuela Moderna se enfrentó a todas las 
normas y creencias establecidas por los poderes eclesiásticos y conservadores, pero también a quienes desde posiciones "revolucionarias" querían suplantar la voz de los obreros y las obreras. Esta escuela tenía el objetivo de crear personas que desarrollaran el razonamiento científico, igualitarias y libres (Solà, 1978). Por ello, no solo consideró que niños y niñas debían ir juntos a las mismas aulas, sino que las diferentes clases sociales, también debían compartirlas. Ferrer i Guàrdia no creía en un tipo de escuela para clases sociales altas y otro para las clases bajas. Partía de que era necesario el contacto entre clases en la escuela para avanzar hacia la igualdad: "La coeducación de pobres y ricos, que pone en contacto unos con otros en la inocente igualdad de la infancia, por medio de la sistemática igualdad de la escuela racional, esa es la escuela, buena, necesaria y reparadora". (Ferrer i Guàrdia, 2000, p. 97).

$\mathrm{Su}$ idea fue hacer un proyecto abiertamente superador de la mediocridad intelectual incorporando las aportaciones de reconocidos intelectuales de muy plurales ideologías y de los movimientos de renovación pedagógica de las escuelas nuevas y las escuelas racionalistas que se habían desarrollado en Europa desde mediados del siglo XIX. Una de las actuaciones que se llevaron a cabo en esta escuela eran las "conferencias dominicales" que tenían el compromiso de facilitar el acceso a la educación y al más alto conocimiento científico y cultural a toda la comunidad. Muchos intelectuales reconocidos colaboraron en ese proyecto, entre ellos Ramón y Cajal. Los domingos se abría la escuela a todas las familias del alumnado para poder participar en estas conferencias y en diversas actividades culturales y educativas. El éxito de las conferencias y los debates llevaron a convertir esta actividad a partir de 1902 en cursos regulares y gratuitos a los que todas las personas interesadas podían participar (Ferrer i Guàrdia, 2000)

La Escuela Moderna es un ejemplo más de cómo los supuestos del Informe Coleman y del modelo de la reproducción estaban equivocados al negar la capacidad de la escuela para transformar las desigualdades.

\section{Actuaciones Educativas de Éxito en la Actualidad: Mejoran el Éxito Escolar y Contribuyen a la Cohesión Social en Todos los Contextos}

Paulo Freire, Ferrer i Guàrdia, y las teorías científicas que a nivel internacional destacan dos factores claves para el aprendizaje en la actual 
sociedad, las interacciones y la participación de la comunidad, están en la base de las actuaciones educativas de éxito. No vamos a entrar a analizarlas aquí en profundidad y a referenciarlas todas. Para ello, se pueden consultar los muchos artículos publicados por las investigadoras y los investigadores que han profundizado en cada una de estas actuaciones como resultado del proyecto de investigación INCLUD-ED: Stretegies for inclusión and social cohesion in Europe from education (2006-2011). INCLUD-ED fue financiado por el $7^{\circ}$ Programa Marco de Investigación de la Unión Europea. La finalidad de esta investigación, desarrollada por investigadoras e investigadores de universidades de 14 países europeos durante cinco años, ha sido crear conocimiento sobre las actuaciones que consiguen el éxito académico y la cohesión social para todos los niños y niñas de todas las comunidades, independientemente de su nivel socioeconómico y origen étnico (Diez, Gatt \& Racionero, 2011; Flecha, García, Gomez \& Latorre, 2009). La investigación estudió el impacto de estas actuaciones en diferentes áreas de la sociedad, como el empleo, la vivienda, la salud y la participación social y política; distinguiendo entre las que obtienen buenos resultados (buenas prácticas) y las actuaciones que obtienen excelentes resultados en muy diversos contextos (actuaciones de éxito) (Valls \& Padros, 2011; Rios, 2013).

Estas actuaciones se han identificado en diversos contextos en los diferentes países de Europa, a través de 20 estudios de caso de prácticas educativas eficaces y 6 estudios de caso longitudinales en los que la comunidad está implicada en proyectos de aprendizaje que han demostrado conseguir excelentes resultados. En todos estos casos había una alta proporción de alumnado de bajo nivel socioeconómico y/o hijos e hijas de inmigrantes o pertenecientes a minorías culturales.

De estas actuaciones mencionamos algunas como los grupos interactivos (organización del aula en pequeños grupos heterogéneos de alumnado con una persona adulta voluntaria en cada uno que promueve la interacción entre los iguales; ninguno es atendido fuera del aula), la lectura dialógica (se realiza en interacción con más personas, en más espacios y tiempos) y la extensión del tiempo de aprendizaje (como los after schools programs, los homework clubs o las bibliotecas tutorizadas). En todas estas actuaciones hay una implicación y participación directa de las familias y de otros miembros de la comunidad, además del profesorado y del alumnado. 


\section{Flecha \& Buslon-Actuaciones Educativas de Éxito}

Recogemos aquí una de las evidencias que demuestran que la implementación de estas actuaciones conlleva un aumento del rendimiento académico sin que ello repercuta en un incremento del coste. Esto es debido a que una de las características es la reorganización de los recursos humanos. Concretamente, en el análisis realizado durante cinco años académicos (del curso 2005-2006 al 2010-2011) en una de las escuelas, situada en uno de los contextos socioeconómicos más desfavorecidos de todos los casos estudiados en INCLUD-ED, donde se aplican las actuaciones educativas de éxito mencionadas se puede observar este hecho. Esta escuela está ubicada en una zona donde se construyeron viviendas de protección oficial en los años noventa y que en el año 2011 acogía a más de un $80 \%$ de población inmigrante, sobretodo de origen magrebí. Ello va acompañado a una situación laboral de gran precariedad que determinaba que el alumnado del centro proviniera de familias con necesidades económicas y laborales severas (Girbés Peco, 2014). A pesar de esta realidad, en cinco años de aplicación de actuaciones educativas de éxito el centro pasó de un $17 \%$ en la adquisición de competencias básicas en lectura a un $85 \%$, ello de forma paralela a que la matrícula del alumnado inmigrante incrementara en un 46\%. Además, en el curso 2010-2011 el alumnado alcanzó el 100\% de la competencia de comprensión oral en inglés situándose por encima de la media de la región. Por lo tanto, el análisis presentado muestra cómo la aplicación de actuaciones educativas de éxito permite mejorar el nivel académico de los alumnos con un uso más eficiente de los recursos (Flecha, 2015).

\section{A Modo de Conclusión}

El Informe Coleman y los estudios análogos condujeron a muchas décadas de análisis del rol de la escuela en la reproducción de las desigualdades sociales. De modo que los planteamientos de Jenks (1972 y 1973) alrededor de las reformas escolares y la igualdad, la sociología inmovilista de Bowles \& Gintis (1976) y la falta de rigurosidad del estructuralismo seguidor de Althusser (1992) repercutieron en la definición científica y política del papel de la educación. Todo ello promovió, como argumentan los científicos sociales de la Black response, planteamientos que se apoyaban en el determinismo socioeconómico y étnico. Como consecuencia se definieron 
posturas y discursos que apostaban por suprimir o debilitar la financiación de programas para salir de la desigualdad, destruían los esfuerzos de profesorado y familias al cumplirse la fatal expectativa y se quitaba todo valor a las ciencias sociales y a la innovación educativa dirigida a la superación de las desigualdades.

A pesar de todas esas décadas del modelo de la reproducción, una gran revolución se está promoviendo desde las ciencias sociales. Los programas de investigación europea, sobretodo del Programa Marco, y probablemente nacionales ya no financiarán ninguna investigación en ciencias sociales que no demuestre que esa inversión tendrá un impacto social, es decir, se revertirá en mejoras concretas para la sociedad.

\section{Referencias}

Althusser, L. (1969). Para leer El Capital. México D.F.: Siglo XXI.

Althusser, L. (1992). El porvenir es largo. Barcelona: Destino.

Apple, M. W. (1986). Teachers and texts. A Political Economy of Class and

Gender Relations in Education. New York: Routledge \& Kegan Paul, Ltd.

Bane, M. J., \& Jencks, C. (1972). The schools and equal opportunity.

Saturday Review: of Education, vol LV, 38, 37-42.

Berstein, B. (1990). Poder, educación y conciencia. Sociología de la transmission cultural. Barcelona: El Roure.

Bowles, S. \& Gintis, H. (1976). Schooling in Capitalist America.

Educational Reform and the contradictions of modern social thought.

New York: Basic Books, Inc. Publishers.

Bowles, S. \& Gintis, H. (1988). Schooling in Capitalist America: Reply to our Critics. Philadelphia: The Falmer Press, Taylor \& Francis.

Coleman, J., Campbell, E., Hobson, C., McPartland, F., Mood, A., Weinfeld, F., et al. (1966). Equality of educational opportunity.

Washington, DC: U.S. Government Printing Office.

Díez, J., Gatt, S., \& Racionero, S. (2011). Placing Immigrant and Minority Family and Community Members at the School's Centre: the role of community participation. European Journal of Education, 46(2), 184-196. doi: 10.1111/j.1465-3435.2011.01474.x 


\section{Flecha \& Buslon-Actuaciones Educativas de Éxito}

Edmonds, R. et al. (1973) A Black Response to Christopher Jenck's Inequality and Certain Other Issues, Harvard Educational Review, 43(1), 76-91.

Ferrer i Guàrdia, F. (2000). La Escuela Moderna. Barcelona: Tusquets.

Flecha, A., García, R., Gómez, A., \& Latorre, A. (2009). Participación en escuelas de éxito: una investigación comunicativa del proyecto Includ-ed. Cultura y educación, 21(2), 183-196.

Flecha, R. (2015). Successful Educational Action for Inclusion and Social Cohesion in Europe. Springer Publishing Company.

Freire, P. (1970). Pedagogía del oprimido. México D.F.: Siglo XXI. Freire, P. (1997). A la sombra de este árbol. Barcelona: El Roure Ciencia. Garcia-Yeste, C., Redondo-Sama, G., Padrós, M. \& Melgar, P. (2016). The Modern School by Francisco Ferrer i Guàrdia (1859-1909): an international and current reference. Teachers College Record, 118(4), $1-36$.

Giddens, A. (1990a). The Consecuences of Modernity. Standford: Standford University Press.

Giddens, A. (1990b). Structuration Theory and Sociological Analysis. In J. Clark, J., C. Modgil \& S. Modgil (Eds.). Anthony Giddens. Consensus and Controversy (pp. 297-315). London: The Falmer Press.

Girbés Peco, S. (2014). El Contrato de Inclusión Dialógica: una actuación de éxito en la superación de la pobreza y la exclusión social en contextos urbanos. Tesis doctoral. Universitat de Barcelona. Disponible en http://diposit.ub.edu/dspace/bitstream/2445/56358/1/SGP_TESIS.pdf

Giroux, H. A. (1992). Border Crossings: Cultural Workers and the Politics of Education. London: Routledge.

Habermas, J. (1987). Teoría de la acción comunicativa. Vol. II. Crítica de la razón funcionalista. Madrid: Taurus.

Jencks, C., et al. (1972). Inequality: A reassessment of the effect of family and schooling in America. New York: Basic Books, Inc., Publishers.

Jencks, C. (1973). Inequality in Retrospect. Harvard Educational Review, 43(1), 138-164.

Jencks, C. (1979). Who Gets Ahead? And inequality: A Comparison. In C. Jencks and others (Eds.). Who Gets Ahead? The Determinants of 
Economic Success in America (pp. 290-311). New York: Basic Books, Inc. Publishers.

Levin, H. (1972). Schooling and inequality: the social science objectivity gap. Saturday Review of Education, 46(11), 49-51.

Rios, O. (2013). Sociocultural transformation \& development. Good practices or successful actions. REMIE Multidisciplinary Journal of Educational Research,3(2), 173. doi: 10.4471/remie.2013.11

Solà, P. (1978). Francesc Ferrer i Guàrdia i l'Escola Moderna. Barcelona: Curial.

Thurow, L. C. (1973). Proving the absence of positive associations.

Harvard Educational Review, 43(1), 106-112.

Valls, R., \& Padrós, M. (2011). Using dialogic research to overcome poverty: From principles to action. European journal of education, 46(2), 173-183. doi: 10.1111/j.1465-3435.2011.01473.x Vygotsky (1979). El desarrollo de los procesos psicológicos superiores. Barcelona: Crítica.

Willis, P. (1990). Common Culture Symbolic Work at Play in the Everyday Cultures of the Young. Boulder: Westview Press.

Ramón Flecha is Professor at Department of Sociology at University of Barcelona.

Nataly Buslon is researcher at Department of Sociology at University of Barcelona.

Contact Address: Direct correspondence to Ramón Flecha, Department of Sociology, University of Barcelona, Dirección: C/Tinent Coronel Valenzuela, 1-11. Facultat d'Economia i Empresa. 08034 Barcelona. E-mail: ramon.flecha@ub.edu 